\title{
Concentration Wave for a Class of Reaction Chromatography System with Pulse Injections
}

\author{
Jing Zhang1, Maofei Shao1, Tao Pan ${ }^{2 *}$ \\ ${ }^{1}$ Department of Mathematics, Jinan University, Guangzhou, China \\ ${ }^{2}$ Department of Optoelectronic Engineering, Jinan University, Guangzhou, China \\ Email: *tpan@jnu.edu.cn
}

Received 19 July 2016; accepted 3 September 2016; published 6 September 2016

Copyright (C) 2016 by authors and Scientific Research Publishing Inc.

This work is licensed under the Creative Commons Attribution International License (CC BY). http://creativecommons.org/licenses/by/4.0/

cC) (7) Open Access

\begin{abstract}
By using fluid dynamics theory with the effects of adsorption and reaction, the chromatography model with a reaction $A \rightarrow B$ was established as a system of two hyperbolic partial differential equations (PDE's). In some practical situations, the reaction chromatography model was simplified a semi-coupled system of two linear hyperbolic PDE's. In which, the reactant concentration wave model was the initial-boundary value problem of a self-closed hyperbolic PDE, while the resultant concentration wave model was the initial-boundary value problem of hyperbolic PDE coupling reactant concentration. The general explicit expressions for the concentration wave of the reactants and resultants were derived by Laplace transform. The $\delta$-pulse and wide pulse injections were taken as the examples to discuss detailedly, and then the stability analysis between the resultant solutions of the two modes of pulse injection was further discussed. It was significant for further analysis of chromatography, optimizing chromatographic separation, determining the physical and chemical characters.
\end{abstract}

\section{Keywords}

Reaction Chromatography Model, Hyperbolic Partial Differential Equations, Initial-Boundary Problem, Stability Analysis

\section{Introduction}

With the appearance of diverse production chromatography (such as the reaction chromatography), the chromato-

"Corresponding author.

How to cite this paper: Zhang, J., Shao, M.F. and Pan, T. (2016) Concentration Wave for a Class of Reaction Chromatography System with Pulse Injections. American Journal of Computational Mathematics, 6, 224-236. 
graphy technology has been widely applied in chemistry, chemical engineering, biological engineering and pharmaceutical engineering, etc., while the demand of chromatography theory is increasing higher. The relationships among the chromatographic input-output and the system conditions play the very important role in chromatography model [1]-[6].

In fact, the mathematical model of chromatography system is a initial-boundary value problem of hyperbolic partial differential equations system [7]-[11], which is hard and challenging mathematics problem to chromatography scientists. In the other hand, the practical application and demand for chromatography is also difficult to understand deeply by mathematicians. The relative works of partial differential equations in the practical chromatography are still not enough.

If the chromatographic process contains reactions, it is labeled as reaction chromatography. An important example is the catalyst for the column packing, accompanied the catalytic [2]-[6] in the adsorption process, and the isomerization reaction is the common situation.

In this paper, a chromatography model with a reaction $A \rightarrow B$ was established, which is a initial-boundary value problem for the semi-coupled system of two linear hyperbolic partial differential equations. Then the general explicit expressions of concentration waves for reactant and resultant were derived using Laplace transform. It was significant for further analysis between input and output of chromatography, optimizing chromatographic separation, determining the physical and chemical characters. Finally, the $\delta$-pulse and wide pulse injections were taken as the examples to discuss detailedly, and then the stability analysis between the resultant solutions of the two modes of pulse injection was further discussed. The results provided proper theory models for further chromatographic data analysis.

\section{Reaction Chromatography Model}

Set the concentrations of the reactant $A$ and the resultant $B$ in the mobile phase and in the stationary phase as $c_{1}, c_{2}, f_{1}, f_{2}$ respectively. Reaction rate was $k_{r}$. And the linear velocity of the mobile phase was $u$. The volume shares in chromatographic column in the mobile phase and in the stationary phase as $\varepsilon, \mu$, respectively.

Denoted that $F=\frac{\mu}{\varepsilon}$, then the mass conservation equations between reactant and resultant in the catalytic chromatographic process was shown as below:

$$
\left\{\begin{array}{l}
\frac{\partial c_{1}}{\partial t}+F \frac{\partial f_{1}}{\partial t}+u \frac{\partial c_{1}}{\partial x}=-k_{r} F f_{1} \\
\frac{\partial c_{2}}{\partial t}+F \frac{\partial f_{2}}{\partial t}+u \frac{\partial c_{2}}{\partial x}=k_{r} F f_{1},
\end{array}\right.
$$

where, $-k_{r} f_{1}$ was the reactant reduction rate, and $k_{r} f_{1}$ was resultant increase rate, $k_{r}$ was the coefficient of reaction rate. According to Langmuir type adsorption isotherms, $f_{1}\left(c_{1}, c_{2}\right)$ and $f_{2}\left(c_{1}, c_{2}\right)$ satisfied for:

$$
\left\{\begin{array}{l}
f_{1}\left(c_{1}, c_{2}\right)=\frac{G_{1} c_{1}}{1+b_{1} c_{1}+b_{2} c_{2}} \\
f_{2}\left(c_{1}, c_{2}\right)=\frac{G_{2} c_{2}}{1+b_{1} c_{1}+b_{2} c_{2}} .
\end{array}\right.
$$

The concentration wave Equation (1) were a system of two nonlinear hyperbolic partial differential equations, which was a hard mathematical problem. But in some practical situations, the problem can be simplified [2]. Assume $c_{1}$ was small, or the adsorption coefficient $b_{1}$ was small, that was, $b_{1} c_{1} \ll 1$. While considering the assumed reaction rate $k_{r}$ is relatively minor, then $c_{2}$ was also small, that was, $c_{2} \ll 1, b_{2} c_{2} \ll 1$. In fact, in the quantitative analysis using high performance liquid chromatography (HPLC), the concentrations of most analytes, such as the reactant $A$ and the resultant $B$ here, were all very small [2] [3]. Thus the adsorption isotherm above can be approximated as a linear and regarded as follows:

$$
f_{1} \doteq G_{1} c_{1}, \quad f_{2} \doteq G_{2} c_{2}
$$

and denoted concretely:

$$
\frac{1+F G_{1}}{u}=\lambda_{1}, \quad \frac{1+F G_{2}}{u}=\lambda_{2}, \frac{k_{r} F G_{1}}{u}=\alpha
$$


they were positive constant, thus Equation (1) can be simplified to the following semi-coupled system of two linear hyperbolic partial differential equations. In which, the reactant concentration wave model was the initialboundary value problem of a self-closed hyperbolic partial differential equations, while the resultant concentration wave model was the initial boundary value problem of hyperbolic partial differential equations coupling reactant concentration.

$$
\left\{\begin{array}{l}
\frac{\partial c_{1}}{\partial x}+\lambda_{1} \frac{\partial c_{1}}{\partial t}=-\alpha c_{1}, \\
\frac{\partial c_{2}}{\partial x}+\lambda_{2} \frac{\partial c_{2}}{\partial t}=\alpha c_{1} .
\end{array}\right.
$$

Chromatographic process started from the boundary, and there were many types of the boundary conditions, such as the injection methods of $\delta$-pulse, wide pulse, head-on, etc.; whose corresponding boundary condition were not zero. The initial state of chromatography columns were typically empty, that the initial conditions corresponding to 0 . However, in practical problems, there were some important chromatograph whose corresponding initial conditions is not zero, such as simulated moving bed chromatography. Therefore, it is necessary to study the general initial-boundary value problem with both the initial and boundary values were not 0 . That was, $c_{1}, c_{2}$ satisfied the following the general initial-boundary value problems.

$$
\begin{aligned}
& \left\{\begin{array}{l}
\frac{\partial c_{1}}{\partial x}+\lambda_{1} \frac{\partial c_{1}}{\partial t}=-\alpha c_{1} \\
c_{1}(x, 0)=c_{1}^{I}(x), \quad 0<x<+\infty \\
c_{1}(0, t)=c_{1}^{B}(t), \quad 0<t<+\infty,
\end{array}\right. \\
& \begin{cases}\frac{\partial c_{2}}{\partial x}+\lambda_{2} \frac{\partial c_{2}}{\partial t}=\alpha c_{1} \\
c_{2}(x, 0)=c_{2}^{I}(x), \quad 0<x<+\infty \\
c_{2}(0, t)=c_{2}^{B}(t), \quad 0<t<+\infty,\end{cases}
\end{aligned}
$$

where, $\lambda_{1}, \lambda_{2}, \alpha$ were constants, $c_{i}^{I}(x), c_{i}^{B}(t), i=1,2$ were positive piecewise and continuous smooth functions, and meet the compatibility condition, $c_{i}^{I}(0)=c_{i}^{B}(0), i=1,2$.

\section{Explicit Solution of Concentration Wave}

Firstly, solved the initial-boundary value problem (6) for concentration wave of of reactant $c_{1}$. According to Laplace transform of $t$, noted that:

$$
L\left[c_{1}(x, t)\right]=\widetilde{c_{1}}(x, p),
$$

it follows from (6) that

$$
\left\{\begin{array}{l}
\frac{\mathrm{d} \tilde{c}_{1}}{\mathrm{~d} x}=-\left(p \lambda_{1}+\alpha\right) \widetilde{c_{1}}+\lambda_{1} c_{1}^{I} \\
\widetilde{c_{1}}(0, p)=\widetilde{c_{1}^{B}}(p) .
\end{array}\right.
$$

Then solved the ordinary differential Equation (8) about $\widetilde{c_{1}}(x, p)$, we got:

$$
\widetilde{c_{1}}(x, p)=\int_{0}^{x} \lambda_{1} c_{1}^{I}(\eta) \mathrm{e}^{\left(p \lambda_{1}+\alpha\right)(\eta-x)} \mathrm{d} \eta+\mathrm{e}^{-\left(p \lambda_{1}+\alpha\right) x} \widetilde{c_{1}^{B}}(p),
$$

and

$$
c_{1}(x, t)=L^{-1}\left[\mathrm{e}^{-\left(p \lambda_{1}+\alpha\right) x} \widetilde{B_{1}^{B}}(p)\right]+L^{-1}\left[\int_{0}^{x} \lambda_{1} c_{1}^{I}(\eta) \mathrm{e}^{\left(p \lambda_{1}+\alpha\right)(\eta-x)} \mathrm{d} \eta\right]
$$

Since 


$$
\begin{gathered}
L^{-1}\left[\mathrm{e}^{-\left(p \lambda_{1}+\alpha\right) x} \widetilde{c_{1}^{B}}(p)\right]=L^{-1}\left[\mathrm{e}^{-\alpha x} L\left[c_{1}^{B}\left(t-\lambda_{1} x\right)\right]\right] \\
= \begin{cases}\mathrm{e}^{-\alpha x} c_{1}^{B}\left(t-\lambda_{1} x\right), & t \geq \lambda_{1} x \\
0, & t<\lambda_{1} x,\end{cases} \\
L^{-1}\left[\int_{0}^{x} \lambda_{1} c_{1}^{I}(\eta) \mathrm{e}^{\left(p \lambda_{1}+\alpha\right)(\eta-x)} \mathrm{d} \eta\right] \\
=\lambda_{1} \int_{0}^{x} c_{1}^{I}(\eta) e^{\left(p \lambda_{1}+\alpha\right)(\eta-x)} \mathrm{d} \eta=L^{-1}\left[\mathrm{e}^{-\alpha x} L\left[c_{1}^{B}\left(t-\lambda_{1} x\right)\right]\right] \\
= \begin{cases}\lambda_{1} \int_{0}^{x} c_{1}^{I}(\eta) \delta\left[t-(x-\eta) \lambda_{1}\right] \mathrm{e}^{\alpha(\eta-x)} \mathrm{d} \eta, & t-(x-\eta) \lambda_{1} \geq 0 \\
0, & t-(x-\eta) \lambda_{1}<0,\end{cases}
\end{gathered}
$$

and

$$
\begin{aligned}
& \lambda_{1} \int_{0}^{x} c_{1}^{I}(\eta) \delta\left[t-(x-\eta) \lambda_{1}\right] \mathrm{e}^{\alpha(\eta-x)} \mathrm{d} \eta \\
& =\int_{0}^{x} c_{1}^{I}(\eta) \delta\left[\eta-\left(x-\frac{t}{\lambda_{1}}\right)\right] \mathrm{e}^{\alpha(\eta-x)} \mathrm{d} \eta= \begin{cases}c_{1}^{I}\left(x-\frac{t}{\lambda_{1}}\right) \mathrm{e}^{-\frac{-\alpha t}{\lambda_{1}}}, & x-\frac{t}{\lambda_{1}} \geq 0 \\
0, & x-\frac{t}{\lambda_{1}}<0 .\end{cases}
\end{aligned}
$$

That is to say,

$$
L^{-1}\left[\int_{0}^{x} \lambda_{1} c_{1}^{I}(\eta) \mathrm{e}^{\left(p \lambda_{1}+\alpha\right)(\eta-x)} \mathrm{d} \eta\right]= \begin{cases}c_{1}^{I}\left(x-\frac{t}{\lambda_{1}}\right) \mathrm{e}^{-\frac{-\alpha t}{\lambda_{1}}}, & t \leq \lambda_{1} x \\ 0, & t>\lambda_{1} x .\end{cases}
$$

To sum (9) and (10) up,

$$
c_{1}(x, t)= \begin{cases}\mathrm{e}^{-\alpha x} c_{1}^{B}\left(t-\lambda_{1} x\right), & t>\lambda_{1} x \\ c_{1}^{I}\left(x-\frac{t}{\lambda_{1}}\right) \mathrm{e}^{-\frac{-\alpha t}{\lambda_{1}}}, & 0<t \leq \lambda_{1} x .\end{cases}
$$

Then solved the initial-boundary value problem (7) for the concentration wave of resultant $c_{2}$. Similarly, according to Laplace transform of $t$, noted that:

$$
L\left[c_{2}(x, t)\right]=\widetilde{c_{2}}(x, p) .
$$

The above problem (7) satisfied the following ordinary differential equation:

$$
\left\{\begin{array}{l}
\frac{\mathrm{d} \widetilde{c_{2}}}{\mathrm{~d} x}=-p \lambda_{2} \widetilde{c_{1}}(x, p)+\lambda_{2} c_{2}^{I}(x)+\alpha \widetilde{c_{1}} \\
\widetilde{c_{2}}(0, p)=\widetilde{c_{2}^{B}}(p) .
\end{array}\right.
$$

Solved the ordinary differential Equation (12) about $\widetilde{c_{2}}(x, p)$, we got:

$$
\widetilde{c_{2}}(x, p)=\int_{0}^{x}\left(\lambda_{2} c_{2}^{I}(\eta)+\alpha \widetilde{c_{1}}\right) \mathrm{e}^{-p \lambda_{2}(x-\eta)} d \eta+\mathrm{e}^{-p \lambda_{2} x} \widetilde{c_{2}^{B}}(p) .
$$

Hence, we got

$$
c_{2}(x, t)=L^{-1}\left[\mathrm{e}^{-p \lambda_{2} x} \widetilde{c_{2}^{B}}(p)\right]+L^{-1}\left[\int_{0}^{x}\left(\lambda_{2} c_{2}^{I}(\eta)+\alpha \widetilde{c_{1}}\right) \mathrm{e}^{p \lambda_{2}(x-\eta)} \mathrm{d} \eta\right]
$$

Since

$$
L^{-1}\left[\mathrm{e}^{-p \lambda_{2} x} \widetilde{c_{2}^{B}}(p)\right]=L^{-1}\left[L\left[c_{2}^{B}\left(t-\lambda_{2} x\right)\right]\right]= \begin{cases}c_{2}^{B}\left(t-\lambda_{2} x\right), & t \geq \lambda_{2} x \\ 0, & t<\lambda_{2} x\end{cases}
$$


and

$$
\begin{aligned}
& L^{-1}\left[\int_{0}^{x}\left(\lambda_{2} c_{2}^{I}(\eta)+\alpha \widetilde{c_{1}}\right) \mathrm{e}^{p \lambda_{2}(x-\eta)} \mathrm{d} \eta\right] \\
&=L^{-1}\left[\int_{0}^{x} \lambda_{2} c_{2}^{I}(\eta) \mathrm{e}^{-p \lambda_{2}(x-\eta)} \mathrm{d} \eta\right]+L^{-1}\left[\int_{0}^{x} \alpha \widetilde{c_{1}} \mathrm{e}^{-p \lambda_{2}(x-\eta)} \mathrm{d} \eta\right], \\
& L^{-1}\left[\int_{0}^{x} \lambda_{2} c_{2}^{I}(\eta) \mathrm{e}^{-p \lambda_{2}(x-\eta)} \mathrm{d} \eta\right]=\int_{0}^{x} \lambda_{2} c_{2}^{I}(\eta) L^{-1}\left[\mathrm{e}^{-p \lambda_{2}(x-\eta)}\right] \mathrm{d} \eta \\
&= \begin{cases}\lambda_{2} \int_{0}^{x} c_{2}^{I}(\eta) \delta\left[t-(x-\eta) \lambda_{2}\right] \mathrm{d} \eta, & \eta \geq x-\frac{t}{\lambda_{2}} \\
0, & \eta<x-\frac{t}{\lambda_{2}},\end{cases}
\end{aligned}
$$

where

$$
\begin{aligned}
\lambda_{2} \int_{0}^{x} c_{2}^{I}(\eta) \delta\left[t-(x-\eta) \lambda_{2}\right] \mathrm{d} \eta & =\int_{0}^{x} c_{2}^{I}(\eta) \delta\left[\eta-\left(x-\frac{t}{\lambda_{2}}\right)\right] \mathrm{d} \eta \\
& = \begin{cases}c_{2}^{I}\left(x-\frac{t}{\lambda_{2}}\right), & 0<t \leq \lambda_{2} x \\
0, & t>\lambda_{2} x .\end{cases}
\end{aligned}
$$

Meanwhile, we had

$$
\begin{aligned}
L^{-1}\left[\int_{0}^{x} \alpha \tilde{c}_{1} \mathrm{e}^{-p \lambda_{2}(x-\eta)} \mathrm{d} \eta\right] & =L^{-1}\left[\int_{0}^{x} L\left[\alpha c_{1}\left(\eta, t-\lambda_{2}(x-\eta)\right)\right] \mathrm{d} \eta\right] \\
& = \begin{cases}\int_{0}^{x} \alpha c_{1}\left(\eta, t-\lambda_{2}(x-\eta)\right) \mathrm{d} \eta, & t-\lambda_{2}(x-\eta) \geq 0 \\
0, & t-\lambda_{2}(x-\eta)<0,\end{cases}
\end{aligned}
$$

and

$$
\int_{0}^{x} \alpha c_{1}\left(\eta, t-\lambda_{2}(x-\eta)\right) \mathrm{d} \eta= \begin{cases}\alpha \int_{x-\frac{t}{\lambda_{2}} c_{1}}^{x}\left(\eta, t-\lambda_{2}(x-\eta)\right) \mathrm{d} \eta, & x-\frac{t}{\lambda_{2}} \geq 0 \\ \alpha \int_{0}^{x} c_{1}\left(\eta, t-\lambda_{2}(x-\eta)\right) \mathrm{d} \eta, & x-\frac{t}{\lambda_{2}}<0 .\end{cases}
$$

To sum (13), (14) and (15) up,

$$
c_{2}(x, t)= \begin{cases}c_{2}^{I}\left(x-\frac{t}{\lambda_{2}}\right)+\alpha \int_{x-\frac{t}{\lambda_{2}}}^{x} c_{1}\left(\eta, t-\lambda_{2}(x-\eta)\right) \mathrm{d} \eta, & 0<t<\lambda_{2} x \\ c_{2}^{B}\left(t-\lambda_{2} x\right)+\alpha \int_{0}^{x} c_{1}\left(\eta, t-\lambda_{2}(x-\eta)\right) \mathrm{d} \eta, & t \geq \lambda_{2} x .\end{cases}
$$

Using the expression (11) of $c_{1}$ and the relation Equation (16) of $c_{1}$ and $c_{2}$, the explicit solution expressions of $c_{2}$ were derived by dividing into the following three cases.

In the case of $\lambda_{1}=\lambda_{2}$, we got

$$
c_{2}(x, t)= \begin{cases}c_{2}^{I}\left(x-\frac{t}{\lambda_{2}}\right)+\left(1-\mathrm{e}^{-\frac{\alpha t}{\lambda_{2}}}\right) c_{1}^{I}\left(x-\frac{t}{\lambda_{2}}\right), & 0<t<\lambda_{2} x \\ c_{2}^{B}\left(t-\lambda_{2} x\right)+c_{1}^{B}\left(t-\lambda_{2} x\right)\left(1-\mathrm{e}^{-\alpha x}\right), & t \geq \lambda_{2} x .\end{cases}
$$

In the case of $\lambda_{1}>\lambda_{2}$, set $y=t-\lambda_{2} x-\left(\lambda_{1}-\lambda_{2}\right) \eta$, then we had 


$$
c_{2}(x, t)=\left\{\begin{array}{cc}
c_{2}^{I}\left(x-\frac{t}{\lambda_{2}}\right)+\frac{\alpha \lambda_{1} \mathrm{e}^{-\frac{\alpha\left(t-\lambda_{2} x\right)}{\lambda_{1}-\lambda_{2}}}}{\lambda_{1}-\lambda_{2}} \int_{x-\frac{t}{\lambda_{2}}}^{x-\frac{t}{\lambda_{1}}} C_{1}^{I}(y) \mathrm{e}^{-\frac{-\alpha \lambda_{2} y}{\lambda_{1}-\lambda_{2}}} \mathrm{~d} y, & 0<t \leq \lambda_{2} x \\
c_{2}^{B}\left(t-\lambda_{2} x\right)+\frac{\alpha \mathrm{e}^{-\frac{\alpha\left(t-\lambda_{2} x\right)}{\lambda_{1}-\lambda_{2}}}}{\lambda_{1}-\lambda_{2}} \int_{0}^{t-\lambda_{2} x} c_{1}^{B}(y) \mathrm{e}^{\frac{\alpha y}{\lambda_{1}-\lambda_{2}}} \mathrm{~d} y & \\
+\frac{\alpha \lambda_{1} e^{-\frac{\alpha\left(t-\lambda_{2} x\right)}{\lambda_{1}-\lambda_{2}}}}{\lambda_{1}-\lambda_{2}} \int_{x-\frac{t}{\lambda_{2}}}^{x-\frac{t}{\lambda_{1}}} c_{1}^{I}(y) \mathrm{e}^{-\frac{\alpha \lambda_{2} y}{\lambda_{1}-\lambda_{2}}} \mathrm{~d} y, & \lambda_{2} x<t \leq \lambda_{1} x \\
c_{2}^{B}\left(t-\lambda_{2} x\right)+\frac{\alpha \mathrm{e}^{-\frac{\alpha\left(t-\lambda_{2} x\right)}{\lambda_{1}-\lambda_{2}}}}{\lambda_{1}-\lambda_{2}} \int_{t-\lambda_{1} x}^{t-\lambda_{2} x} c_{1}^{B}(y) \mathrm{e}^{\frac{\alpha y}{\lambda_{1}-\lambda_{2}}} \mathrm{~d} y, & t \geq \lambda_{1} x .
\end{array}\right.
$$

In the case of $\lambda_{1}<\lambda_{2}$, we had $y=t-\lambda_{2} x-\left(\lambda_{1}-\lambda_{2}\right) \eta$, then we got

$$
c_{2}(x, t)=\left\{\begin{array}{cc}
c_{2}^{I}\left(x-\frac{t}{\lambda_{2}}\right)+\frac{\alpha \lambda_{1} \mathrm{e}^{-\frac{\alpha\left(t-\lambda_{2} x\right)}{\lambda_{1}-\lambda_{2}}}}{\lambda_{1}-\lambda_{2}} \int_{x-\frac{t}{\lambda_{2}}}^{x-\frac{t}{\lambda_{1}}} c_{1}^{I}(y) \mathrm{e}^{-\frac{\alpha \lambda_{2} y}{\lambda_{1}-\lambda_{2}}} \mathrm{~d} y, & 0<t \leq \lambda_{1} x \\
c_{2}^{I}\left(x-\frac{t}{\lambda_{2}}\right)-\frac{\alpha \mathrm{e}^{-\frac{\alpha\left(t-\lambda_{2} x\right)}{\lambda_{1}-\lambda_{2}}}}{\lambda_{1}-\lambda_{2}} \int_{0}^{t-\lambda_{1} x} c_{1}^{B}(y) \mathrm{e}^{\frac{\alpha y}{\lambda_{1}-\lambda_{2}}} d y & \\
-\frac{\alpha \lambda_{1} e^{-\frac{\alpha\left(t-\lambda_{2} x\right)}{\lambda_{1}-\lambda_{2}}}}{\lambda_{1}-\lambda_{2}} \int_{0}^{x-\frac{t}{\lambda_{2}}} C_{1}^{I}(y) \mathrm{e}^{-\frac{\alpha \lambda_{2} y}{\lambda_{1}-\lambda_{2}}} \mathrm{~d} y, & \lambda_{1} x<t \leq \lambda_{2} x \\
c_{2}^{B}\left(t-\lambda_{2} x\right)+\frac{\alpha \mathrm{e}^{-\frac{\alpha\left(t-\lambda_{2} x\right)}{\lambda_{1}-\lambda_{2}}}}{\lambda_{1}-\lambda_{2}} \int_{t-\lambda_{1} x}^{t-\lambda_{2} x} c_{1}^{B}(y) \mathrm{e}^{\frac{\alpha y}{\lambda_{1}-\lambda_{2}}} \mathrm{~d} y, & t \geq \lambda_{2} x .
\end{array}\right.
$$

Particularly, when the initial-boundary problem (6) and (7) satisfied the following conditions

$$
c_{1}(x, 0)=c_{2}(x, 0)=c_{2}(0, t)=0
$$

the explicit solution of reactant and resultant concentration wave $c_{1}, c_{2}$ were obtained as follows.

Following (11), we had

$$
c_{1}(x, t)= \begin{cases}\mathrm{e}^{-\alpha x} c_{1}^{B}\left(t-\lambda_{1} x\right), & t>\lambda_{1} x \\ 0, & 0<t \leq \lambda_{1} x .\end{cases}
$$

According to the expressions (17), (18) and (19), we had the explicit solution expressions of $c_{2}$ as follows. When $\lambda_{1}=\lambda_{2}$

$$
c_{2}(x, t)= \begin{cases}0, & 0<t<\lambda_{2} x \\ c_{1}^{B}\left(t-\lambda_{2} x\right)\left(1-\mathrm{e}^{-\alpha x}\right), & t \geq \lambda_{2} x .\end{cases}
$$

When $\lambda_{1}>\lambda_{2}$

$$
c_{2}(x, t)= \begin{cases}0, & 0<t \leq \lambda_{2} x \\ \frac{\alpha \mathrm{e}^{-\frac{\alpha\left(t-\lambda_{2} x\right)}{\lambda_{1}-\lambda_{2}}}}{\lambda_{1}-\lambda_{2}} \int_{0}^{t-\lambda_{2} x} c_{1}^{B}(y) \mathrm{e}^{\frac{\alpha y}{\lambda_{1}-\lambda_{2}}} \mathrm{~d} y, & \lambda_{2} x<t \leq \lambda_{1} x \\ \frac{\alpha \mathrm{e}^{-\frac{\alpha\left(t-\lambda_{2} x\right)}{\lambda_{1}-\lambda_{2}}}}{\lambda_{1}-\lambda_{2}} \int_{t-\lambda_{1} x}^{t-\lambda_{2} x} c_{1}^{B}(y) \mathrm{e}^{\frac{\alpha y}{\lambda_{1}-\lambda_{2}}} \mathrm{~d} y, & t \geq \lambda_{1} x .\end{cases}
$$


When $\lambda_{1}<\lambda_{2}$

$$
C_{2}(x, t)= \begin{cases}0, & 0<t \leq \lambda_{1} x \\ -\frac{\alpha \mathrm{e}^{-\frac{\alpha\left(t-\lambda_{2} x\right)}{\lambda_{1}-\lambda_{2}}}}{\lambda_{1}-\lambda_{2}} \int_{0}^{t-\lambda_{1} x} c_{1}^{B}(y) \mathrm{e}^{\frac{\alpha y}{\lambda_{1}-\lambda_{2}}} \mathrm{~d} y, & \lambda_{1} x<t \leq \lambda_{2} x \\ \frac{\alpha \mathrm{e}^{-\frac{\alpha\left(t-\lambda_{2} x\right)}{\lambda_{1}-\lambda_{2}}}}{\lambda_{1}-\lambda_{2}} \int_{t-\lambda_{1} x}^{t-\lambda_{2} x} c_{1}^{B}(y) \mathrm{e}^{\frac{\alpha y}{\lambda_{1}-\lambda_{2}}} \mathrm{~d} y, & t \geq \lambda_{2} x .\end{cases}
$$

\section{Solutions and Stability for $\delta$-Pulse and Wide Pulse Injections}

In this section, we derived the solutions of reactant and resultant concentration waves in wide pulse and $\delta$-pulse injections detailedly. And the stability analysis between the resultant solutions of the two modes of pulse injection was further discussed.

\section{1. $\delta$-Pulse Injection}

Chromatographic process started from the boundary, and there were many types of the boundary conditions, such as the methods of $\delta$-pulse, wide pulse, head-on, etc; whose corresponding boundary condition was not zero. Where, $\delta$-pulse and wide pulse were the most common way of chromatography injection method. Firstly, initial state of chromatography column in the $\delta$-pulse method, which injection function was a kind of $\delta$-function, was typically empty. So in the case of $\delta$-Pulse, $c_{1}$ satisfied the following initial-boundary problem.

$$
\left\{\begin{array}{l}
\frac{\partial c_{1}}{\partial x}+\lambda_{1} \frac{\partial c_{1}}{\partial t}=-\alpha c_{1} \\
c_{1}(x, 0)=0, \quad 0<x<+\infty \\
c_{1}(0, t)=k \delta(t), \quad 0<t<+\infty
\end{array}\right.
$$

where $k$ is a constant represented the injection size, which is equal to $c_{10} t_{p}$ in wide pulse method in Section 4.2. According to the behavior of the $\delta$-function, we had

$$
\begin{gathered}
\int_{0}^{\infty} \delta(t) \mathrm{d} t=1, \\
\int_{0}^{\infty} C_{1}(0, t) \mathrm{d} t=\int_{0}^{\infty} k \delta(t) \mathrm{d} t=k .
\end{gathered}
$$

The solution of concentration wave for reactant was obtained by Laplace transform as similar with Section 3. The concentration wave corresponding to $\delta$-pulse injection of reactant and resultant can be expressed as follows.

$$
c_{1}(x, t)= \begin{cases}k \mathrm{e}^{-\alpha x} \delta\left(t-\lambda_{1} x\right), & t \geq \lambda_{1} x \\ 0, & t<\lambda_{1} x .\end{cases}
$$

If there was no reaction terms, that was, $\alpha=0$, we got

$$
c_{1}(x, t)= \begin{cases}k \delta\left(t-\lambda_{1} x\right), & t \geq \lambda_{1} x \\ 0, & t<\lambda_{1} x .\end{cases}
$$

As for the solution of concentration wave for resultant, the initial and boundary values were both 0 . From the expression (21), (22) and (23), we had the explicit solution expressions of $c_{2}$.

When $\lambda_{1}=\lambda_{2}$

$$
c_{2}(x, t)= \begin{cases}0, & 0<t<\lambda_{2} x \\ k \delta\left(t-\lambda_{2} x\right)\left(1-\mathrm{e}^{-\alpha x}\right), & t \geq \lambda_{2} x .\end{cases}
$$

It was equivalent to $c_{2}(x, t)=0$. 
When $\lambda_{1}>\lambda_{2}$

$$
c_{2}(x, t)= \begin{cases}0, & 0<t \leq \lambda_{2} x \\ \frac{\alpha k}{\lambda_{1}-\lambda_{2}} \mathrm{e}^{-\frac{\alpha\left(t-\lambda_{2} x\right)}{\lambda_{1}-\lambda_{2}}}, & \lambda_{2} x<t \leq \lambda_{1} x \\ 0, & t \geq \lambda_{1} x .\end{cases}
$$

When $\lambda_{1}<\lambda_{2}$

$$
c_{2}(x, t)= \begin{cases}0, & 0<t \leq \lambda_{1} x \\ \frac{\alpha k}{\lambda_{2}-\lambda_{1}} \mathrm{e}^{-\frac{\alpha\left(t-\lambda_{2} x\right)}{\lambda_{1}-\lambda_{2}}}, & \lambda_{1} x<t \leq \lambda_{2} x \\ 0, & t \geq \lambda_{2} x .\end{cases}
$$

\subsection{Wide Pulse Injection}

Wide pulse was the another most common way of chromatography injection method, its initial state of chromatography column was typically empty, so the initial condition was the follows,

$$
c_{1}^{I}(x) \equiv 0, \quad c_{2}^{I}(x) \equiv 0 .
$$

The corresponding injection function was given as follows,

$$
c_{1}^{B}(t)=\left\{\begin{array}{ll}
c_{10}, & 0<t \leq t_{p} \\
0, & t_{p}<t,
\end{array} \quad c_{2}^{B}(x) \equiv 0 .\right.
$$

where, $t_{p}$ was the injection time, $c_{10}$ was the injection rate, both of them are constant. In this paper, Wide pulse was taken as an another example, the solution of concentration wave for reactant and resultant were derived detailedly.

Similarly, we had the explicit solution expressions of $c_{1}$ and $c_{2}$ as follows,

$$
c_{1}(x, t)= \begin{cases}0, & t \leq \lambda_{1} x \\ c_{10} \mathrm{e}^{-\alpha x}, & \lambda_{1} x<t \geq t_{p}+\lambda_{1} x, \\ 0, & t_{p}+\lambda_{1} x<t .\end{cases}
$$

When $\lambda_{1}=\lambda_{2}$,

$$
c_{2}(x, t)= \begin{cases}0, & 0<t<\lambda_{2} x \\ c_{10}\left(1-\mathrm{e}^{-\alpha x}\right), & \lambda_{2} x<t \leq t_{p}+\lambda_{2} x \\ 0, & t_{p}+\lambda_{2} x<t .\end{cases}
$$

When $\lambda_{1}>\lambda_{2}$, we got,

$$
C_{2}(x, t)= \begin{cases}0, & 0<t \leq \lambda_{2} x \\ c_{10}\left(1-\mathrm{e}^{-\frac{\alpha\left(t-\lambda_{2} x\right)}{\lambda_{1}-\lambda_{2}}}\right), & \lambda_{2} x<t \leq \lambda_{1} x, t \leq t_{p}+\lambda_{2} x \\ c_{10} \mathrm{e}^{-\frac{\alpha\left(t-\lambda_{2} x\right)}{\lambda_{1}-\lambda_{2}}\left(\mathrm{e}^{\frac{\alpha t_{p}-\lambda_{2}}{\lambda_{1}}}-1\right),} & \lambda_{2} x<t \leq \lambda_{1} x, t>t_{p}+\lambda_{2} x \\ c_{10}\left(1-\mathrm{e}^{-\alpha x}\right), & \lambda_{1} x<t \leq t_{p}+\lambda_{2} x \\ c_{10}\left(\mathrm{e}^{\frac{\alpha\left(t_{p}+\lambda_{2} x-t\right)}{\lambda_{1}-\lambda_{2}}}-\mathrm{e}^{-\alpha x}\right), & t>\lambda_{1} x, t_{p}+\lambda_{2} x<t \leq t_{p}+\lambda_{1} x \\ 0, & t>t_{p}+\lambda_{1} x .\end{cases}
$$


When $\lambda_{1}<\lambda_{2}$,

$$
C_{2}(x, t)= \begin{cases}0, & 0<t \leq \lambda_{1} x \\ c_{10}\left(\mathrm{e}^{-\frac{\alpha\left(\lambda_{2} x-t\right)}{\lambda_{2}-\lambda_{1}}}-\mathrm{e}^{-\alpha x}\right), & \lambda_{1} x<t \leq \lambda_{2} x, t \leq t_{p}+\lambda_{1} x \\ c_{10} \mathrm{e}^{-\frac{\alpha\left(\lambda_{2} x-t\right)}{\lambda_{2}-\lambda_{1}}}\left(1-\mathrm{e}^{-\frac{\alpha t_{p}}{\lambda_{2}-\lambda_{1}}}\right), & \lambda_{1} x<t \leq \lambda_{2} x, t>t_{p}+\lambda_{1} x \\ C_{10}\left(1-\mathrm{e}^{-\alpha x}\right), & \lambda_{2} x<t \leq t_{p}+\lambda_{1} x \\ c_{10}\left(1-\mathrm{e}^{-\frac{\alpha\left(t_{p}+\lambda_{2} x-t\right)}{\lambda_{2}-\lambda_{1}}}\right), & t>\lambda_{2} x, t_{p}+\lambda_{1} x<t \leq t_{p}+\lambda_{2} x \\ 0, & t>t_{p}+\lambda_{2} x .\end{cases}
$$

\subsection{Stability Analysis between Wide Pulse and $\delta$-Pulse Injections}

Note that, the boundary condition in wide pulse injection tended to the condition in $\delta$-pulse injection. We also showed that the mentioned limit relationship was still valid for the solutions in the two modes of pulse injection. The main result of this work is the following theorem:

Theorem 1. If $t_{p} \rightarrow 0, c_{10} \rightarrow \infty$ and $t_{p} c_{10} \rightarrow k$, the solution of concentration wave for resultant in wide pulse injection converges to the resultant solution in $\delta$-pulse injection.

Proof. 1) When $\lambda_{1}=\lambda_{2}$, from (32), For any fixed $x_{0}$, when $t \in\left(0, \lambda_{2} x_{0}\right]$, we had

$$
c_{2}(x, t)=0 \text {, }
$$

and when $t \in\left(\lambda_{2} x_{0}, \infty\right), \exists t_{p}$ (a sufficiently small constant), so that $t_{p}+\lambda_{2} x_{0}<t$, and

$$
c_{2}\left(x_{0}, t, t_{p}\right)=0 .
$$

By arbitrariness of $x_{0}$, we obtained

$$
\lim _{\substack{t_{p} \rightarrow 0 \\ c_{10} \rightarrow+\infty \\ t_{p} c_{10} \rightarrow B}} c_{2}\left(x, t, t_{p}\right)=0,
$$

which was converging to the solution (27) in $\delta$-pulse injection.

2) When $\lambda_{1}>\lambda_{2}$, the resultant solution (33) in wide pulse injection can be expressed as follows.

In the case of $0<x \leq \frac{t_{p}}{\lambda_{1}-\lambda_{2}}$,

$$
c_{2}(x, t)= \begin{cases}0, & 0<t \leq \lambda_{2} x \\ c_{10}\left(1-\mathrm{e}^{\left.-\frac{\alpha\left(t-\lambda_{2} x\right)}{\lambda_{1}-\lambda_{2}}\right),}\right. & \lambda_{2} x<t \leq \lambda_{1} x \\ C_{10}\left(1-\mathrm{e}^{-\alpha x}\right), & \lambda_{1} x<t \leq t_{p}+\lambda_{2} x \\ C_{10}\left(\mathrm{e}^{\frac{\alpha\left(t_{p}+\lambda_{2} x-t\right)}{\lambda_{1}-\lambda_{2}}}-\mathrm{e}^{-\alpha x}\right), & t_{p}+\lambda_{2} x<t \leq t_{p}+\lambda_{1} x \\ 0, & t_{p}+\lambda_{1} x<t .\end{cases}
$$

In the case of $\frac{t_{p}}{\lambda_{1}-\lambda_{2}}<x$, 


$$
C_{2}(x, t)= \begin{cases}0, & 0<t \leq \lambda_{2} x \\ c_{10}\left(1-\mathrm{e}^{\left.-\frac{\alpha\left(t-\lambda_{2} x\right)}{\lambda_{1}-\lambda_{2}}\right),}\right. & \lambda_{2} x<t \leq t_{p}+\lambda_{2} x \\ C_{10} \mathrm{e}^{-\frac{\alpha\left(t-\lambda_{2} x\right)}{\lambda_{1}-\lambda_{2}}\left(\mathrm{e}^{\frac{\alpha t_{p}-\lambda_{2}}{\lambda_{1}}}-1\right),} & t_{p}+\lambda_{2} x<t \leq \lambda_{1} x \\ C_{10}\left(\mathrm{e}^{\frac{\alpha\left(t_{p}+\lambda_{2} x-t\right)}{\lambda_{1}-\lambda_{2}}}-\mathrm{e}^{-\alpha x}\right), & \lambda_{1} x<t \leq t_{p}+\lambda_{1} x \\ 0, & t_{p}+\lambda_{1} x<t .\end{cases}
$$

For any fixed $x_{0}, \exists t_{p}$ (a sufficiently small constant), so that $x_{0}>\frac{t_{p}}{\lambda_{1}-\lambda_{2}}$. Then expressions (37) can be noted to:

$$
c_{2}\left(x_{0}, t, t_{p}\right)= \begin{cases}0, & 0<t \leq \lambda_{2} x_{0} \\ c_{10}\left(1-\mathrm{e}^{-\frac{\alpha\left(t-\lambda_{2} x_{0}\right)}{\lambda_{1}-\lambda_{2}}}\right), & \lambda_{2} x_{0}<t \leq t_{p}+\lambda_{2} x_{0} \\ c_{10} \mathrm{e}^{-\frac{\alpha\left(t-\lambda_{2} x_{0}\right)}{\lambda_{1}-\lambda_{2}}\left(\mathrm{e}^{\frac{\alpha t_{p}-\lambda_{2}}{\lambda_{1}}}-1\right),} & t_{p}+\lambda_{2} x_{0}<t \leq \lambda_{1} x_{0} \\ c_{10}\left(\mathrm{e}^{\frac{\alpha\left(t_{p}+\lambda_{2} x_{0}-t\right)}{\lambda_{1}-\lambda_{2}}}-\mathrm{e}^{-\alpha x_{0}}\right), & \lambda_{1} x_{0}<t \leq t_{p}+\lambda_{1} x_{0} \\ 0, & t_{p}+\lambda_{1} x_{0}<t .\end{cases}
$$

Furthermore, for any fixed $t_{0}$,

a) When $t \in\left(0, \lambda_{2} x_{0}\right]$, we had

$$
c_{2}\left(x, t_{0}, t_{p}\right)=0 .
$$

b) When $t \in\left(\lambda_{2} x_{0}, \lambda_{1} x_{0}\right], \exists t_{p}$ (a sufficiently small constant), $t_{p}+\lambda_{2} x_{0}<t \leq \lambda_{1} x_{0}$, we got

$$
c_{2}\left(x_{0}, t_{0}, t_{p}\right)=c_{10} \mathrm{e}^{-\frac{\alpha\left(t-\lambda_{2} x_{0}\right)}{\lambda_{1}-\lambda_{2}}}\left(\mathrm{e}^{\frac{\alpha t_{p}}{\lambda_{1}-\lambda_{2}}}-1\right) .
$$

and

$$
\begin{aligned}
\lim _{\substack{t_{p} \rightarrow 0 \\
c_{10} \rightarrow+\infty \\
t_{p} c_{10} \rightarrow B}} c_{2}\left(x_{0}, t_{0}, t_{p}\right) & =\lim _{\substack{t_{p} \rightarrow 0 \\
c_{10} \rightarrow+\infty \\
t_{p} c_{10} \rightarrow B}} c_{10} \mathrm{e}^{-\frac{\alpha\left(t_{0}-\lambda_{2} x_{0}\right)}{\lambda_{1}-\lambda_{2}}}\left(\mathrm{e}^{\frac{\alpha t_{p}}{\lambda_{1}-\lambda_{2}}}-1\right) \\
& =\frac{\alpha B}{\lambda_{1}-\lambda_{2}} \mathrm{e}^{-\frac{\alpha\left(t_{0}-\lambda_{2} x_{0}\right)}{\lambda_{1}-\lambda_{2}}} .
\end{aligned}
$$

c) When $t \in\left(\lambda_{1} x_{0}, \infty\right], \exists t_{p}$ (a sufficiently small constant), $t_{p}+\lambda_{1} x_{0}<t_{0}$, we had

$$
\lim _{\substack{t_{p} \rightarrow 0 \\ c_{10} \rightarrow+\infty \\ t_{p} c_{10} \rightarrow B}} c_{2}\left(x_{0}, t_{0}, t_{p}\right)=0 .
$$


To sum up,

$$
\lim _{\substack{t_{p} \rightarrow 0 \\ c_{10} \rightarrow+\infty \\ t_{p} c_{10} \rightarrow B}} c_{2}\left(x, t, t_{p}\right)= \begin{cases}0, & 0<t \leq \lambda_{2} x \\ \frac{\alpha B}{\lambda_{1}-\lambda_{2}} \mathrm{e}^{-\frac{\alpha\left(t-\lambda_{2} x\right)}{\lambda_{1}-\lambda_{2}}}, & \lambda_{2} x<t \leq \lambda_{1} x \\ 0 & \lambda_{1} x<t .\end{cases}
$$

3) When $\lambda_{1}<\lambda_{2}$, the solution in wide pulse method (34) was equivalent to the following.

In the case of $0<x \leq \frac{t_{p}}{\lambda_{2}-\lambda_{1}}$,

$$
c_{2}(x, t)= \begin{cases}0, & 0<t \leq \lambda_{1} x \\ c_{10}\left(\mathrm{e}^{\frac{\alpha\left(t-\lambda_{2} x\right)}{\lambda_{2}-\lambda_{1}}}-\mathrm{e}^{-\alpha x}\right), & \lambda_{1} x<t \leq \lambda_{2} x \\ c_{10}\left(1-\mathrm{e}^{-\alpha x}\right), & \lambda_{2} x<t \leq t_{p}+\lambda_{1} x \\ c_{10}\left(1-\mathrm{e}^{\frac{\alpha\left(t_{p}+\lambda_{2} x-t\right)}{\lambda_{1}-\lambda_{2}}}\right), & t_{p}+\lambda_{1} x<t \leq t_{p}+\lambda_{2} x \\ 0, & t_{p}+\lambda_{2} x<t .\end{cases}
$$

In the case of $\frac{t_{p}}{\lambda_{2}-\lambda_{1}}<x$,

$$
C_{2}(x, t)= \begin{cases}0, & 0<t \leq \lambda_{1} x \\ c_{10}\left(\mathrm{e}^{\frac{\alpha\left(t-\lambda_{2} x\right)}{\lambda_{2}-\lambda_{1}}}-\mathrm{e}^{-\alpha x}\right), & \lambda_{1} x<t \leq t_{p}+\lambda_{1} x \\ C_{10} \mathrm{e}^{\frac{\alpha\left(t-\lambda_{2} x\right)}{\lambda_{2}-\lambda_{1}}}\left(1-\mathrm{e}^{\frac{\alpha t_{p}}{\lambda_{1}-\lambda_{2}}}\right), & t_{p}+\lambda_{1} x<t \leq \lambda_{2} x \\ c_{10}\left(1-\mathrm{e}^{\frac{\alpha\left(t_{p}+\lambda_{2} x-t\right)}{\lambda_{1}-\lambda_{2}}}\right), & \lambda_{2} x<t \leq t_{p}+\lambda_{2} x \\ 0, & t_{p}+\lambda_{2} x<t .\end{cases}
$$

For any fixed $x_{0}, \exists t_{p}$ (a sufficiently small constant), so that $x_{0}>\frac{t_{p}}{\lambda_{2}-\lambda_{1}}$. Then expression (41) can be noted to:

$$
c_{2}\left(x_{0}, t, t_{p}\right)= \begin{cases}0, & 0<t \leq \lambda_{1} x_{0} \\ c_{10}\left(\mathrm{e}^{\frac{\alpha\left(t-\lambda_{2} x_{0}\right)}{\lambda_{2}-\lambda_{1}}}-\mathrm{e}^{-\alpha x_{0}}\right), & \lambda_{1} x_{0}<t \leq t_{p}+\lambda_{1} x_{0} \\ c_{10} \mathrm{e}^{\frac{\alpha\left(t-\lambda_{2} x_{0}\right)}{\lambda_{2}-\lambda_{1}}}\left(1-\mathrm{e}^{\frac{\alpha t_{p}}{\lambda_{1}-\lambda_{2}}}\right), & t_{p}+\lambda_{1} x_{0}<t \leq \lambda_{2} x_{0} \\ c_{10}\left(1-\mathrm{e}^{\left.\frac{\alpha\left(t_{p}+\lambda_{2} x_{0}-t\right)}{\lambda_{1}-\lambda_{2}}\right)},\right. & \lambda_{2} x_{0}<t \leq t_{p}+\lambda_{2} x_{0} \\ 0, & t_{p}+\lambda_{2} x_{0}<t,\end{cases}
$$


and for any $t_{0}$,

i) When $t \in\left(0, \lambda_{1} x_{0}\right]$, we had

$$
c_{2}\left(x, t_{0}, t_{p}\right)=0 .
$$

ii) When $t \in\left(\lambda_{1} x_{0}, \lambda_{2} x_{0}\right], \exists t_{p}$ (a sufficiently small constant), $t_{p}+\lambda_{1} x_{0}<t \leq \lambda_{2} x_{0}$, we had

$$
\begin{gathered}
c_{2}\left(x_{0}, t_{0}, t_{p}\right)=c_{10} \mathrm{e}^{\frac{\alpha\left(t_{0}-\lambda_{2} x_{0}\right)}{\lambda_{2}-\lambda_{1}}}\left(1-\mathrm{e}^{\frac{\alpha t_{p}}{\lambda_{1}-\lambda_{2}}}\right) . \\
\lim _{\substack{t_{p} \rightarrow 0 \\
c_{10} \rightarrow+\infty \\
t_{p} c_{10} \rightarrow B}} c_{2}\left(x_{0}, t_{0}, t_{p}\right)=\lim _{\substack{t_{p} \rightarrow 0 \\
c_{10} \rightarrow \infty \\
t_{p} c_{10} \rightarrow B}} c_{10} \mathrm{e}^{\frac{\alpha\left(t_{0}-\lambda_{2} x_{0}\right)}{\lambda_{2}-\lambda_{1}}}\left(1-\mathrm{e}^{\frac{\alpha t_{p}}{\lambda_{1}-\lambda_{2}}}\right)=\frac{\alpha B}{\lambda_{2}-\lambda_{1}} \mathrm{e}^{\frac{\alpha\left(t_{0}-\lambda_{2} x_{0}\right)}{\lambda_{2}-\lambda_{1}}} .
\end{gathered}
$$

iii) When $t \in\left(\lambda_{2} x_{0}, \infty\right], \exists t_{p}$ (a sufficiently small constant), $t_{p}+\lambda_{2} x_{0}<t_{0}$, we had

$$
\lim _{\substack{t_{p} \rightarrow 0 \\ c_{10} \rightarrow+\infty \\ t_{p} c_{10} \rightarrow B}} c_{2}\left(x_{0}, t_{0}, t_{p}\right)=0 .
$$

By arbitrariness of $x_{0}$ and $t_{0}$, we obtained

$$
\lim _{\substack{t_{p} \rightarrow 0 \\ c_{10} \rightarrow+\infty \\ t_{p} c_{10} \rightarrow B}} c_{2}\left(x, t, t_{p}\right)= \begin{cases}0, & 0<t \leq \lambda_{2} x \\ \frac{\alpha B}{\lambda_{2}-\lambda_{1}} \mathrm{e}^{-\frac{\alpha\left(t-\lambda_{2} x\right)}{\lambda_{1}-\lambda_{2}}}, & \lambda_{2} x<t \leq \lambda_{1} x \\ 0 & \lambda_{1} x<t .\end{cases}
$$

By (35), (39) and (43), we can conclude that this Theorem is true.

\section{Conclusion}

The chromatography model with a reaction $A \rightarrow B$ was established and can be simplified a semi-coupled system of two linear hyperbolic PDE's in some practical situations. In which, the reactant concentration wave model was the initial-boundary value problem of a self-closed hyperbolic PDE, while the resultant concentration wave model was the initial-boundary value problem of hyperbolic PDE coupling reactant concentration. The general explicit expressions for the concentration wave of the reactants and resultants were derived by Laplace transform. The $\delta$-pulse and wide pulse injections were taken as the examples to discuss detailedly, and it was proved that the continuous dependence of solutions was in accordance with the dependence under corresponding boundary conditions. It was significant for further analysis of chromatography in nonlinear case, optimizing chromatographic separation, determining the physical and chemical characters.

\section{Acknowledgements}

This work was supported by National Natural Science Foundation of China (No. 21312045) and Science and Technology Project of Guangdong Province of China (No. 2014A020213016).

\section{References}

[1] Guiochon, G., Ghodbane, S., Golshan-Shirazi, S., et al. (1989) Non-Linear Chromatography: Recent Theoretical and Experimental Results. Talanta, 36, 19-33. http://dx.doi.org/10.1016/0039-9140(89)80079-7

[2] Lin, B. (2004) Guiding of the Chromatography Model Theory. Science Press, Beijing.

[3] Lin, B., Song, F. and Guiochon, G. (2003) Analytical Solution of Ideal Nonlinear Model of Reaction Chromatography for a Reaction $A \rightarrow B$ and a Parabolic Isotherm. Journal of Chromatography A, 1003, 91-100. http://dx.doi.org/10.1016/S0021-9673(03)00656-3

[4] Qamar, S., Perveen, S. and Seidel-Morgenstern, A. (2016) Analysis of a Two-Dimensional Nonequilibrium Model of Linear Reactive Chromatography Considering Irreversible and Reversible Reactions. Industrial \& Engineering Chemi- 
stry Research, 55, 2471-2482. http://dx.doi.org/10.1021/acs.iecr.5b04714

[5] Bibi, S., Qamar, S. and Seidel-Morgenstern, A. (2015) Irreversible and Reversible Reactive Chromatography: Analytical Solutions and Moment Analysis for Rectangular Pulse Injections. Journal of chromatography, 1385, 49-62. http://dx.doi.org/10.1016/j.chroma.2015.01.065

[6] Qamar, S., Bibi, S., Khan, F.U., Shah, M., Javeed, S. and Seidel-Morgenstern, A. (2014) Irreversible and Reversible Reactions in a Liquid Chromatographic Column: Analytical Solutions and Moment Analysis. Industrial \& Engineering Chemistry Research, 53, 2461-2472. http://dx.doi.org/10.1021/ie403645w

[7] Pan, T. and Lin, L. (1995) The Global Solution of the Scalar Nonconvex Conservation Laws with Boundary Condition. Journal of Partial Differential Equations, 8, 371-383.

[8] Pan, T, Liu, H. and Nishihara, K. (1999) Asymptotic Stability of the Rarefaction Wave of a One Dimensional Model System for Compressible Viscous Gas with Boundary. Japan Journal Industrial Applied Mathematics, 16, 431-441. http://dx.doi.org/10.1007/BF03167367

[9] Pan, T. and Jiu, Q. (1999) Asymptotic Behavior for Solution of the Scalar Viscous Conservation Laws in the Bounded Interval Corresponding to Rarefaction Waves. Progress in Natural Science, 9, 948-952.

[10] Pan, T. and Liu, H. (2002) Asymptotic Behaviors of the Solution to an Initial-boundary Value Problem for Scalar Viscous Conservation Laws. Applied Mathematics Letters, 15, 727-734. http://dx.doi.org/10.1016/S0893-9659(02)00034-4

[11] Pan, T., Liu, H. and Nishihara, K. (2002) Asymptotic Behavior of a One-Dimensional Compressible Viscous Gas with Free Boundary. SIAM Journal on Mathematical Analysis, 34, 273-291. http://dx.doi.org/10.1137/S0036141001385745

\section{Submit or recommend next manuscript to SCIRP and we will provide best service for you:}

Accepting pre-submission inquiries through Email, Facebook, LinkedIn, Twitter, etc.

A wide selection of journals (inclusive of 9 subjects, more than 200 journals)

Providing 24-hour high-quality service

User-friendly online submission system

Fair and swift peer-review system

Efficient typesetting and proofreading procedure

Display of the result of downloads and visits, as well as the number of cited articles

Maximum dissemination of your research work

Submit your manuscript at: http://papersubmission.scirp.org/ 